\title{
From Stochastic Grammar to Bayes Network: Probabilistic Parsing of Complex Activity
}

\author{
Nam N. Vo and Aaron F. Bobick \\ Georgia Institute of Technology \\ namvodgatech.edu, afbecc.gatech.edu
}

\begin{abstract}
We propose a probabilistic method for parsing a temporal sequence such as a complex activity defined as composition of sub-activities/actions. The temporal structure of the high-level activity is represented by a string-length limited stochastic context-free grammar. Given the grammar, a Bayes network, which we term Sequential Interval Network (SIN), is generated where the variable nodes correspond to the start and end times of component actions. The network integrates information about the duration of each primitive action, visual detection results for each primitive action, and the activity's temporal structure. At any moment in time during the activity, message passing is used to perform exact inference yielding the posterior probabilities of the start and end times for each different activity/action. We provide demonstrations of this framework being applied to vision tasks such as action prediction, classification of the high-level activities or temporal segmentation of a test sequence; the method is also applicable in Human Robot Interaction domain where continual prediction of human action is needed.
\end{abstract}

\section{Introduction}

For a variety of activity monitoring tasks ranging from surveillance to work-flow monitoring to quality control inspection, the challenge is to observe some complex activity being performed and to be able to label which action has been performed and often to parse or segment the input sequence into its component actions. Additionally, if a system is intended to respond appropriately and at the correct time with respect to an activity, it is necessary to perform such parsing while the activity is occurring; examples of this last task are seen in the domain of human robot interaction $[9,10]$.

In this paper we consider the problem of parsing complex activities where we recursively define such activities to be compositions of some combination of complex (sub)activities and primitive actions. We presume as given the

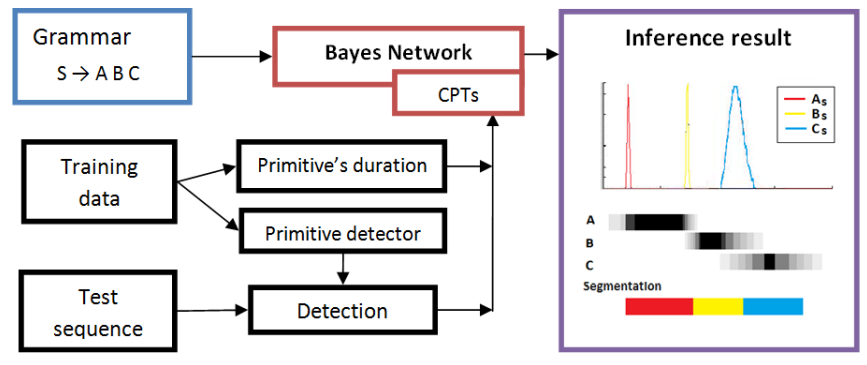

Figure 1. Overview of our framework

temporal structure and decomposition of the activity, such as a task plan of an assembly process where there may be partially ordered or even optional steps. We further assume that probabilistic low level visual detectors are provided or learned from training examples; these detectors provide noisy evidence as to occurrence of some primitive action. The goal is to develop a probabilistic representation of such activities that leverage the temporal constraints and local sensing and which allows the system to assess, at any moment in time, the probability as to which actions have occurred or will occur and when.

The overview of our framework is shown in Figure 1. First, a stochastic-context-free-grammar is used to represent the activitys structure: AND operations in the grammars production rules encode ordered composition of subactions while the OR operations permit variation in the course of actions. Next we develop a method to generate the Sequential Interval Network (SIN), a discrete-time graphical model whose hidden nodes are actions timings, observed nodes are the output of primitive actions detectors, and edges encodes dependencies between action-action or action-detection. Given the networks conditional probability tables (CPT) computed using the learned duration models and the visual detector outputs, we describe how to perform exact inference by a message-passing algorithm. Our framework has several advantages: (1) The grammar offers a hierarchical representation of the activity, allowing multiple layers of abstraction when defining action compositions (2) The primitive actions detector is assumed to be a black- 
box and can be engineered independent of the grammar. (3) The information obtained from the inference output is rich: the posterior probability of whether an action happens and if so when it happens. The probability of an action being active at a time step can also be inferred for every action and every time step both in the past and arbitrarily far in the future.

The following section discusses related works. In Section 3, we describe the grammar notation. Detail of the generation and inference on SIN are shown in Section 4. We present several experiments in Section 5 and 6. We conclude and discuss future works in Section 7.

\section{Related Works}

Broadly, previous work in activity recognition can be characterized as belonging to one of two classes of analysis. The first focuses on recognizing short, low-level actions. Common approaches use BOW framework with video feature such as STIP, HOG3D, Cuboid [24], Dense Trajectories [23]. When these short time actions appear in a sequence of such primitives, there is the more challenging tasks of segmenting the sequence or localizing the actions within the sequence. For example in [19, 8], SVM is used for classification and segmentation is chosen to maximize the total SVM score using dynamic programming. Amer et al [2] use Sum Product Network and Tang et al [22] use hidden semi-markov model (HSMM) to exploit temporal structure for action recognition and event detection. Both leverage BOW feature representation.

The second type of activity representation and recognition methods considers more complex activities that can be meaningfully decomposed into smaller components. A variety of models have been proposed to represent the compositional structure and to perform the video analysis. An popular line of approaches is to detect a sequence of discrete events from the raw visual input, and then apply string parsing technique. In $[11,16]$, traditional stochastic context free grammar parsing algorithm was adapted for Computer Vision problems; adjustments were made to handle different types of errors. To the same end, Damen et al [4] proposed Attribute Multiset Grammars which can encode richer constraints on activity structure. For parsing, an automatically generated Bayesian Network is used to find the detections that correspond to the best explanation. Albanese et al [1] used Probabilistic Petri Net to detect interesting human activities. In [15], Probabilistic Suffix Tree was used to learn the pattern of symbols (or "actionlets") for early detection of on-going activity. Similarly in [21, 18], AND-OR grammar is learnt from example strings of symbols, each represents an action according to the grammar's language. The learnt grammar discovers the pattern of actions and can be used for prediction. This, however, assumes that the string is not noisy i.e. the primitive action detectors are accurate.
Different from these approaches, we assume detection of primitive actions is not a discrete set of events, but more general: a "heatmap" that represents the action likelihood for every interval, hence our framework can principally handle wide range of detection uncertainties.

The approaches most related to our work are Dynamic Bayes Network (DBN) methods [20, 14] in which the system's state encodes when each action starts and ends. Inference by a particle filter is done in streaming mode. While the current state can be inferred, it is computationally infeasible to derive the distribution of the start and end of actions at arbitrary points in the past or future (prediction) using all available observation up till the current time. Koppula et al [13] introduce Anticipatory Temporal Conditional Random Field, which is an undirected graphical model designed to run online like a DBN. Prediction is done by extending the network into the future. Most recently, Wei et al [25] proposed method for modeling sequence that could incorporate rich information (duration and object/human poses) about each action. However, these approaches have to resort to approximate inference since it is infeasible to explore every possible state of every timesteps. Our framework can be considered a class of segment model and HSMM [17, 26]. By reasoning on the timings, it has the similar rich capability while permitting exact inference.

\section{Modeling complex activity structure by a Stochastic Grammar}

The complex composite activity can be represented in hierarchical fashion: the activity consists of several actions, which can in turn consist of even smaller actions and so on. Therefore we define two types of actions: the action that is represented as a composition of other actions, and the primitive action which is explicitly modeled using learned duration model and visual detector. The whole activity is the top-level composition.

We use a stochastic grammar to model this hierarchical structure. The grammar is formally defined as $\mathrm{G}=(\mathrm{S}, \mathrm{T}$, $\mathrm{N}, \mathrm{R}$ ) where: $\mathrm{T}$ is the set of terminal symbols, corresponding to the primitives, $\mathrm{N}$ is the set of non-terminal symbols, corresponding to the compositions, $\mathrm{S}$ is the starting symbol, corresponding to the top-level activity, $\mathrm{R}$ is the set of probabilistic production rules, which define the compositions. The stochastic component of the grammar is reflected in the probabilities associated with these production rules.

\subsection{Compile the grammar}

Before generating SIN, a preprocessing step is necessary to convert the original grammar to a "compiled version" that satisfies three constraints. Two of these constraints are merely syntactic and do not restrict the structure of the top level activity. The last constraint places a string-length limitation that bounds the length of time it takes to complete 


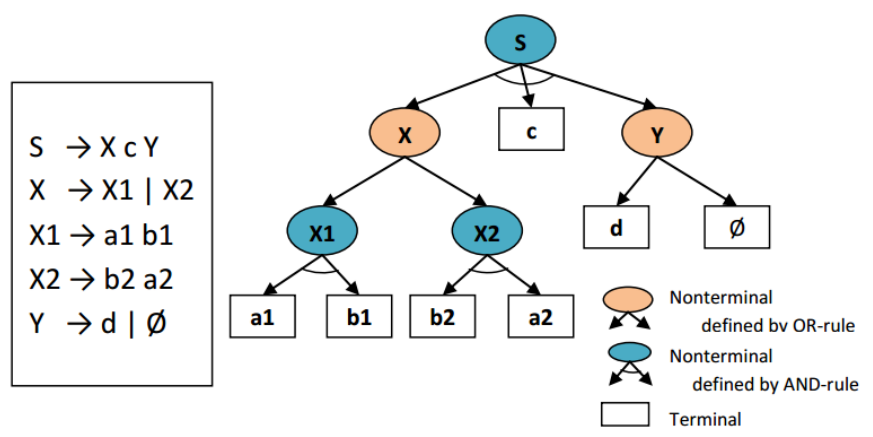

(a)

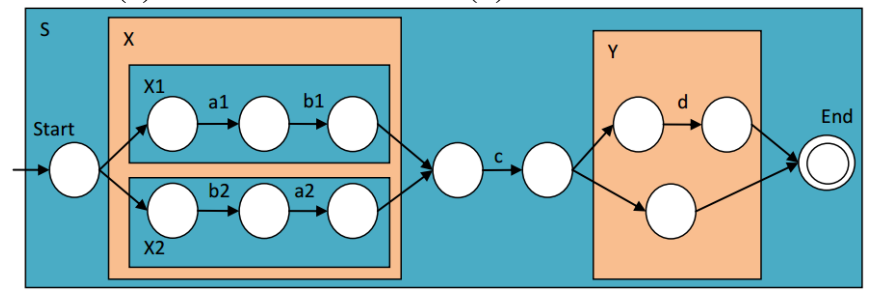

(c)

Figure 2. Example activity: "S $S \rightarrow(a b \mid b a) c(d \mid \emptyset) "$. Probabilities associated with the OR-rules were not shown in this figure. Other equivalent representations: (a) the compiled grammar, (b) the AND-OR tree, (c) Acyclic Finite State Machine

the activity.

1. Each production rule must be either an AND-rule or an OR-rule. Mixing of AND and OR operations in one rule is not permitted. However, such rule can be trivially converted to several pure AND-rules and ORrules. Note that since the grammar is stochastic, each symbol on the right hand side of the OR-rule is associated with a probability and they sum to 1 .

2. Every symbol can only appear on the right hand side of a production rule at most once. That is every copy of a single action that appears in more than one rule must be a distinct instance. However, these instances will share detectors and duration models (described later) making the system no more difficult to implement.

3. The grammar cannot generate arbitrary long strings since our Bayes network will cover all possible sequences. This means rules causing loop such as: " $S \rightarrow$ $S A \mid A$ " are not allowed. Explicitly unrolling such loops to a maximum number of times can be done to avoid this situation.

An example grammar is show in Figure 2.a. The toplevel activity is a partially ordered sequence of the actions $\mathrm{a}, \mathrm{b}$ in any order, followed by action c, ending with an optional action d. Figure 2.b displays the AND-OR tree of the grammar.

\section{Sequential Interval Network (SIN)}

We describe how to generate the network that will reason about timings in sequential data. Our input will be the compiled grammar of the activity. First we define random variables $A_{s}$ and $A_{e}$ representing the starting time and ending time for every action $A$, and let $Z^{A}$ be the observations of the detector associated with action $\mathrm{A}$; we describe $Z^{A}$ shortly. Our formulation is defined on a discrete and bounded representation of time where $1 \leq A_{s} \leq A_{e} \leq T$, where $T$ is defined to be large enough to cover all variations in the activitys length. Depending on the course of actions as permitted by the grammar, action $A$ may happen or it may not. We employ the special value -1 to denote the case when action $A$ does not happen. We will use the notations $(\exists A)$ and $(! A)$ to stand for the case $A$ happens $\left(1 \leq A_{s} \leq A_{e} \leq T\right)$ and $A$ does not happen $\left(A_{s}=A_{e}=-1\right)$.

We now design a network that includes nodes $A_{s}, A_{e}$ and observations $Z^{A}$ for every symbol $A$ in the grammar. The basic idea is that SIN is constructed in a hierarchical fashion, similar to the AND-OR tree (Figure 2.b). To do so, we describe how to construct it recursively for the three cases of action primitives, AND-rules, and OR-rules. We then show a recursive message passing algorithm to perform exact inference on the constructed network; the output of the inference are the posterior distributions of the start and the end of every action $P\left(A_{s} \mid Z\right), P\left(A_{e} \mid Z\right)$ including the possibility that the action does not occur $\left(A_{s}=A_{e}=-1\right)$.

\subsection{The primitive $v$}

The portion of the network that corresponds to a primitive $v$ is shown in Figure 3.a. We use the notation $Z^{\text {pre }(A)}$ and $Z^{\text {post }(A)}$ to stand for the observation of all actions that happen before $A$ and after $A$, respectively. There are two conditional probabilities required for this component:

The condition probability $P\left(v_{e} \mid v_{s}\right)$ : represents the prior information about the duration of action $v$. In our implementation we define: $P\left(v_{e} \mid v_{s}\right) \propto N\left(v_{e}-v_{s} ; \mu_{v}, \sigma_{v}\right)$ if $v_{e}-v_{s} \geq d \min _{v}$, or 0 otherwise, where $N(. ;$.$) is$ the Gaussian density function and $\mu_{v}, \sigma_{v}$ are parameters learned from labeled training data. Note that the Gaussian is truncated to avoid too small (or even negative) duration. For the special case when the action does not happen the duration is defined as: $P\left(v_{e}=-1 \mid v_{s}=-1\right)=1$.

Likelihood $P\left(Z^{v} \mid v_{s}, v_{e}\right)$ : each primitive has a visual detector that outputs a detection score $F_{v}[\alpha, \beta]$ representing the evidence that the action starts at time $\alpha$ and ends at time $\beta$ for every possible interval $(\alpha, \beta)$ of the range [1, $\mathrm{T}]$ (covering the entire activity). Then the likelihood can be computed based on that detection: $P\left(Z^{v} \mid v_{s}=\alpha\right.$, v.e $=$ $\beta)=h_{v} F_{v}[\alpha, \beta]$ for some constant $h_{v}$. Calculation of $F_{v}$ can be assumed to be a black box procedure.

We also need to define the likelihood for the special 


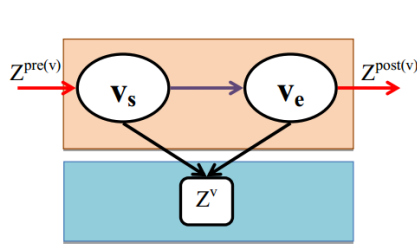

(a)

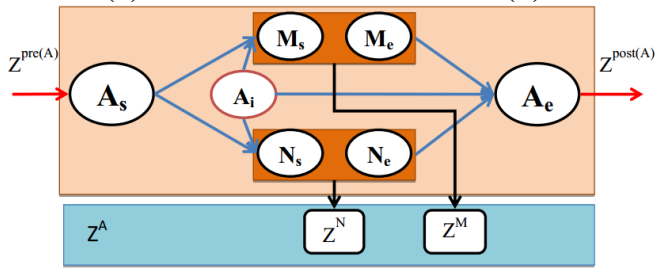

(c)

Figure 3. Structure of the network: (a) primitive v, (b) composition A defined by AND-rule $\mathrm{A} \rightarrow \mathrm{MN}$, (c) composition A defined by OR-rule $\mathrm{A} \rightarrow \mathrm{M} \mid \mathrm{N}$

case $P\left(Z^{v} \mid v_{s}=-1, v_{e}=-1\right)$ which can be written as $P\left(Z^{v} \mid ! v\right)=h_{v} F_{v}[-1,-1]$. We assign to $F_{v}[-1,-1]$ a "null value" defined as the expected detection score (Alternatively if the detector can detect if the action does not happen, it can be incorporated into this likelihood).

\subsection{The composition defined by AND-rule $A \rightarrow M N$}

This rule defines the action $A$ to be the sequence of subaction $M$ and $N$ (possibly more). The network is shown in Figure 3.b. Here we make some important assumptions: (1) the start and end of the composition are the start of the first action and the end of the last action in the sequence respectively $\left(A_{s}=M_{s}, A_{e}=N_{e}\right)$, (2) the end of one action is equal the start of the next action in the sequence $\left(N_{s}=M_{e}\right),{ }^{1}$ (3) the observation of the action consists of all observations of its sub-actions $Z^{A}=Z^{M, N}$

\subsection{The OR-rule composition $A \rightarrow M \mid N$}

Figure 3.c shows the OR network structure. The OR-rule defines a composite action $A$ to be either $M(\exists M$ and $! N$, which means $\left.A_{s}=M_{s}, A_{e}=M_{e}, N_{s}=N_{e}=-1\right)$ or $N\left(\exists N\right.$ and $! M$, which means $A_{s}=N_{s}, A_{e}=N_{e}, M_{s}=$ $\left.M_{e}=-1\right)$.

The standard approach to realizing this "OR" condition in a Bayes network is to use the multiplexer CPT, with a "selector" variable [12] which we write as $A_{i}$ in our network. $A_{i} \in\{M, N\}$ indicate which case it is $(\exists M$ or $\exists N)$. The prior probability $P\left(A_{i} \mid \exists A\right)$, or equivalently $P(\exists M \mid \exists A)$ and $P(\exists N \mid \exists A)$, is extracted from the grammar's production rule. Note that it can be manually defined or learned from training data (usually we will choose $P\left(A_{i}=M \mid \exists A\right)=P\left(A_{i}=N \mid \exists A\right)=0.5$, unless otherwise stated).

\footnotetext{
${ }^{1}$ If time between actions is needed we can insert a special DUMMY action between them.
}

Finally for every composition $\mathrm{A}$, we can define $P\left(Z^{A} \mid ! A\right)=\prod_{\mathrm{M} \text { in A }} P\left(Z^{M} \mid ! M\right)$. Note that scaling the likelihood $P\left(Z^{v} \mid v_{s}, v_{e}\right)$ does not affect the final result. Therefore in the implementation we could choose the scaling such that $h_{v} F_{v}[-1,-1]=1$ for every primitive $\mathrm{v}$, then we can safely ignore the factors $P\left(Z^{A} \mid ! A\right)$ for every A.

\subsection{Exact inference by message passing}

Given the constructed network, we now compactly show a 4-step inference process (a more explicit description will be given in the supplemental document). Beside the CPT $P\left(v_{e} \mid v_{s}\right)$ and $P\left(Z^{v} \mid v_{s}, v_{e}\right)$ described in section 4.1, we need three more inputs: (1) The prior $P(\exists S)$, (2) $P\left(S_{s} \mid \exists S\right)$ : the constraint about the start, and (3) $P\left(Z^{\text {end }} \mid S_{e}, \exists S\right)$ : the constraint about the end. We set $P(\exists S)=1$ to make following formulation simple (though rule such as "S $\rightarrow A \mid \emptyset "$ can be used to emulate the case where the activity does not happen all together). For the task of activity segmentation, we can have: the start is the first time step/frame and the end is the last time step/frame of the test sequence (experiment in section 6). On the other hand, we can assume uniform distributions about the start and the end of the activity (experiment in section 5). In that case, our framework effectively performs detection and parsing at the same time.

Step 1 - Forward phase: Starting from $P\left(S_{s} \mid \exists S\right)$, the propagation is performed from the high level actions to their subactions recursively, from the start of the action to the end, integrating all observations in the process. The output is $P\left(A_{s}, Z^{\text {pre }(A)} \mid \exists A\right), P\left(A_{e}, Z^{\text {pre }(A), A} \mid \exists A\right)$ for every action $\mathrm{A}$.

For primitive v, given $P\left(v_{s}, Z^{\text {pre }(v)} \mid \exists v\right)$ : we can multiply it with the duration factor $P\left(v_{e} \mid v_{s}\right)$ and visual observation factor $P\left(Z^{v} \mid v_{s}, v_{e}\right)$ to get $P\left(v_{s}, v_{e}, Z^{\text {pre }(v), v} \mid \exists v\right)$. Then marginalization can be done to get $P\left(v_{e}, Z^{\text {pre }(v), v} \mid \exists v\right)$.

For AND-rule $A \rightarrow M N$ : given $P\left(A_{s}, Z^{\text {pre }(A)} \mid \exists A\right)$, the variable $M_{s}$ has the same distribution. Recursively perform forward phase on $\mathrm{M}$ to get $P\left(M_{e}, Z^{\text {pre }(A), M} \mid \exists M\right)$. Next the variable $N_{s}$ has the same distribution, so we can perform forward phase on $\mathrm{N}$ to get $P\left(N_{e}, Z^{p r e}(A), M, N \mid \exists N\right)$. The final result is the same as distribution $P\left(A_{e}, Z^{\operatorname{pre}(A), A} \mid \exists A\right)$.

For OR-rule $A \rightarrow M \mid N$ : given $P\left(A_{s}, Z^{\text {pre }(A)} \mid \exists A\right)$, we can represent the distribution of $A_{e}$ in term of $M_{e}$ and $N_{e}$. For example if $\mathrm{A}$ is $\mathrm{M}$, then the distribution $P\left(M_{s}, Z^{\operatorname{pre}(M)} \mid \exists M\right)$ is the same as $A_{s}$, we can therefore perform forward phase on $\mathrm{M}$ to get $P\left(M_{e}, Z^{M, p r e(M)} \mid \exists M\right)$. Similarly we can find 
$P\left(N_{e}, Z^{N, p r e(N)} \mid \exists N\right)$, then distribution of $A_{e}$ is:

$$
\begin{aligned}
& P\left(A_{e}=\beta, Z^{A, p r e(A)} \mid \exists A\right)= \\
& P(\exists M \mid \exists A) P\left(Z^{N} \mid ! N\right) P\left(M_{e}=\beta, Z^{M, p r e(M)} \mid \exists M\right)+ \\
& \quad P(\exists N \mid \exists A) P\left(Z^{M} \mid ! M\right) P\left(N_{e}=\beta, Z^{N, p r e(N)} \mid \exists N\right)
\end{aligned}
$$

Step 2 - Backward phase: Similarly, starting from $P\left(Z^{\text {end }} \mid S_{e}, \exists S\right)$, we compute $P\left(Z^{\text {post }(A)} \mid A_{e}, \exists A\right)$ and $P\left(Z^{A, p o s t}(A) \mid A_{s}, \exists A\right)$ for every action A (propagation in the opposite direction to the first step).

Step 3 - Compute the posteriors: by multiplying forward and backward messages, we get $P\left(A_{s}, Z \mid \exists A\right)$, $P\left(A_{e}, Z \mid \exists A\right)$ for every action $\mathrm{A}$. If $\mathrm{v}$ is a primitive, we can have the joint distribution:

$P\left(v_{s}, v_{e}, Z \mid \exists v\right)=P\left(v_{s}, v_{e}, Z^{\text {pre }(v), v} \mid \exists v\right) P\left(Z^{\text {post }(v)} \mid v_{e}, \exists v\right)$ Also we can find $P(Z)=\sum_{t} P\left(S_{s}=t, Z\right)$

Step 4 - Compute the happening probability: starting with $P(\exists S \mid Z)=P(\exists S)=1$, we find $P(\exists A \mid Z)$ for every action A recursively.

For AND-rule $A \rightarrow M N$ : given $P(\exists A \mid Z)$, then $P(\exists M \mid Z)=P(\exists N \mid Z)=P(\exists A \mid Z)$

For OR-rule $A \rightarrow M \mid N$ : given $P(\exists A \mid Z)$, we compute (apply similar formulas for $\mathrm{N}$ ):

$$
\begin{gathered}
P(\exists M, Z \mid \exists A)=P(\exists M \mid \exists A) \sum_{t>0} P\left(M_{e}=t, Z \mid \exists M\right) \\
P(\exists M \mid Z)=P(\exists A \mid Z) \frac{P(\exists M, Z \mid \exists A)}{P(\exists M, Z \mid \exists A)+P(\exists N, Z \mid \exists A)}
\end{gathered}
$$

Output: the probability of action A happening $P(\exists A \mid Z)$, and if that is the case, the distribution of the start and the end $P\left(A_{s}, Z \mid \exists A\right), P\left(A_{e}, Z \mid \exists A\right)$, or even the joint of them if $\mathrm{A}$ is a primitive.

\subsection{Interpreting the result for recognition, detec- tion/prediction and segmentation}

First if a symbol $\mathrm{A}$ is on the right hand side of an ORrule, then $P(\exists A \mid Z)$ is the posterior probability associated with that OR-rule. Hence we can do action recognition and infer the most probable course of actions.

Secondly we can compute $P\left(A_{s} \mid Z\right), P\left(A_{e} \mid Z\right)$ :

$$
P\left(A_{s} \mid Z\right)=P(\exists A \mid Z) \frac{P\left(A_{s}, Z \mid \exists A\right)}{\sum_{t>0} P\left(A_{s}=t, Z \mid \exists A\right)}
$$

for values between 1 and $\mathrm{T}$ (note that $P\left(A_{s}=-1 \mid Z\right)=$ $P(! A \mid Z)=1-P(\exists A \mid Z))$. These distributions are shown in the experiment in section 5. Using these distributions, prediction of when an action starts or ends can be made by picking the expected value, or the value that maximize the posterior. Even better, one could consume this whole distribution to account for the inferred uncertainty depending on specific application.
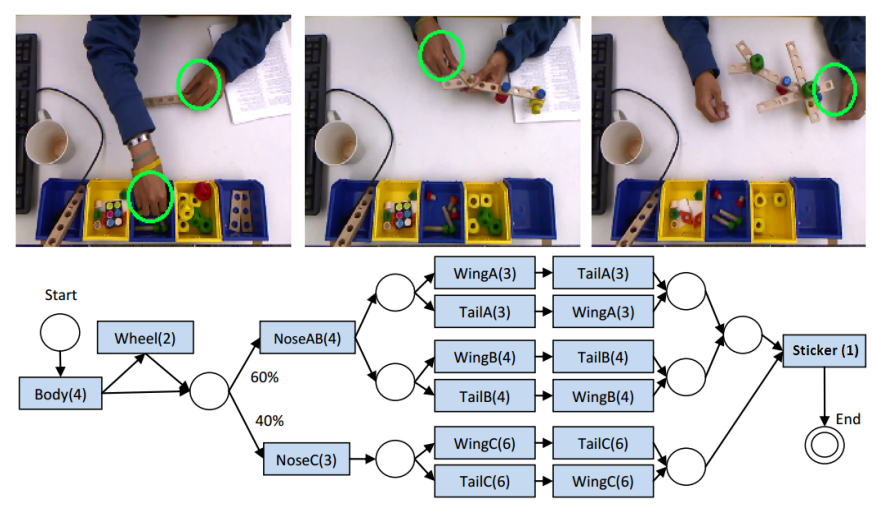

Figure 4. The toy assembly task. (a) Example input frames and hand detection result. (b) The temporal structure in the form of state machine. Each box is an AND composition of a number of primitives (shown in the bracket). The subject first assembles the body part; follow by the optional wheel part and one of the two nose parts. Then the wing part and tail part can be done in any order. Finally the subject puts the sticker on the model. There are 40 different primitive actions and 12 complete task variations.

These results can be mapped back to the original grammar: to compute the distribution of actions' timing in the original grammar, one can combine the distributions of separate actions in the compiled version corresponding to the same action in the original version.

For the task of labeling frames, the probability of a time step $t$ having the label of primitive $v$ can be computed easily:

$$
P\left(\text { label }_{t}=v \mid Z\right)=\sum_{\alpha=1}^{t} \sum_{\beta=t}^{T} P\left(v_{s}=\alpha, v_{e}=\beta \mid Z\right)
$$

We obtain the distribution of the label of time step t. If A is a composition, $P\left(\right.$ label $\left._{t}=A \mid Z\right)$ can be found be summing over all its subactions. Therefore temporal segmentation can be done in any hierarchy level by choose the label that maximize the probability. We perform activity segmentation in term of primitives in the experiments to demonstrate this feature. Alternative method of segmentation is to derive the parsing with highest probability (the most probable course of actions), estimates the start and the end of the actions in that sequence, and then labels the frames.

\subsection{Implementation Explanation}

The primitive detector is the only component that processes the test sequence and it is particularly important. Not only does it affect the action localization result, it impacts the OR-rule situations. For example given $A \rightarrow M \mid N$, a strong detection of subactions in $M$ can make $P(\exists M \mid Z)$ higher, while diminishing $P(\exists N \mid Z)$ at the same time.

We assume this procedure is a black-box so that making use of different kinds of detectors is possible. Note that the calculation $P\left(Z^{v} \mid v_{s}=\alpha, v_{e}=\beta\right) \propto F_{v}[\alpha, \beta]$ can leverage all the observation data available, not just the seg- 

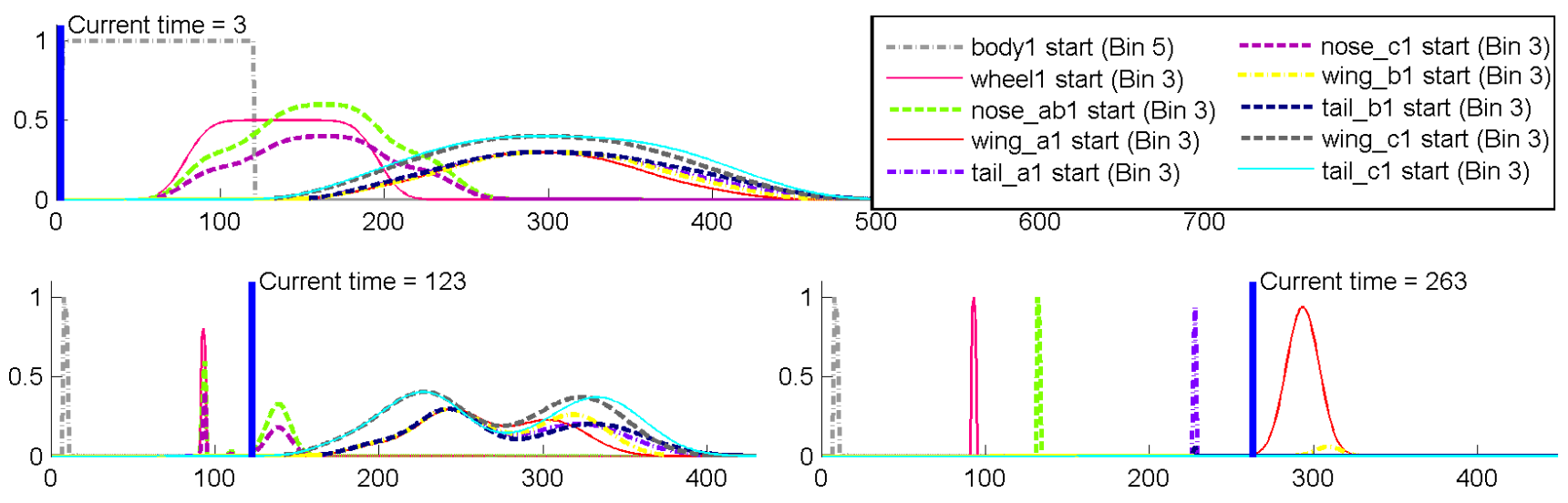

Figure 5. (best view in color) Example posterior distributions of the starts of some actions (first primitive of each part) at 3 different time steps (computed using Eq.3, note that the special value (-1) is not shown). In order to observe them easily, each distributions is scaled so that its peak have the same value as the probability that action would happen (e.g. for body 1 , it is 1 ; for nose_ab1, it's 0.6 in the first timestep and about 0.99 in the last timestep; for nose_c1, it's 0.4 and then 0.01 )

ment $[\alpha, \beta]$. If it is a likelihood based detector, then the score can be used directly. If it is a discriminative method, then a post-processing step to calibrate the score is needed (because each detector might output different kinds of confidence scores). For example one can normalize the SVMscore and apply a sigmoid function, or apply a exponential function to the negative of the energy-score in order to obtain a score that better indicates the likelihood. The likelihood value 0 is usually discouraged as it could nullify the likelihood of other actions.

Computational complexity: The inference complexity is linear in number of nodes (number of actions in the grammar: K) and the size of CPT $\left(T^{2}\right)$, i.e. $O\left(K . T^{2}\right)$.

Parsing in streaming mode: This can be done by constructing the entire network at the beginning, with all likelihoods initialized using the expected detection score (the "null value" $\left.F_{v}[-1,-1]\right)$. As new observations are obtained, likelihoods are recomputed and the inference process is re-performed.

\section{Toy assembly task experiment}

To demonstrate the capability of SIN, we designed a simple toy assembly task, where the human operator takes wooden pieces provided in 5 different bins in the workspace and puts them together to construct an airplane model. The tasks structure is shown in Figure 4 and the subjects will follow this recipe. Our dataset consists of 29 sequences; each one is about 2-3 minutes long. Results are generated by performing 20 trials where on each trial 3 sequences (1 of each airplane model A, B and C) are randomly selected for testing and the remaining are for training.

Each primitive action is defined as getting a piece from a bin and assembling it. The start of the action is defined as when the hand reaches the piece. In order to detect such actions, first we implement a simple color blob detector to

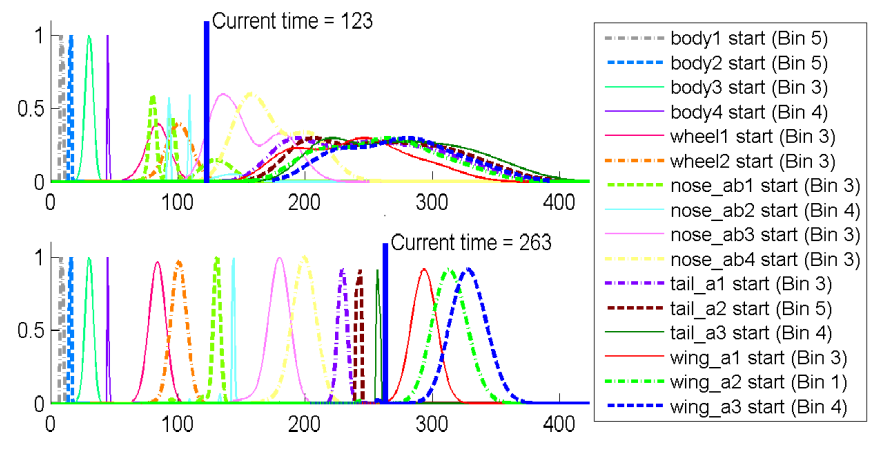

Figure 6. In this example, detectors of actions reaching for pieces in bin 3 are disabled. Here we show all actions that actually happen in this sequence.

detect the hand positions in the input frame (note that its performance is not extremely accurate: it fails to to detect the correct hands roughly $30 \%$ of the time). Then we can compute $F_{v}[\alpha, \beta] \propto N\left(H_{\alpha} ; \mu_{v}, \sigma_{v}\right)+u_{v}$, where $H_{t}$ is the position of the hands at frame t, $\mathrm{N}(. ;)$ is the Gaussian density function and parameters $\mu_{v}, \sigma_{v}$ are learned, and $u_{v}$ is a small uniform component representing the likelihood in case the hand detector fails. Notice that: (1) in this case the action detectors reduce to special case: event detectors of the actions starts; (2) actions corresponding to different pieces in the same bin will end up having similar detectors (our method naturally resolves this ambiguity).

Qualitative Result: in Figure 5, some example posterior distribution outputs when running our method on a sequence in streaming mode are shown (we encourage readers to watch the supplementary video). At first no observation is available; the distributions are determined by the prior information about the start of the task (which we set to be a uniform in first 30s) and duration models of primitives. In the second plot, some first actions (Body and Nose) are detected; however it is still not clear which model is being 
done, hence the distributions of all possible actions overlap both in the past and future. Finally, these uncertainties are resolved when the subject is about to finish TailA part. It is recognized that model $\mathrm{A}$ is being assembled and the next actions going to be WingA; distributions of model B and C's actions have all diminished. As we can see as time progresses: (1) ambiguities both in the past and future are resolved, (2) the distributions get tighter, hence the prediction of when the actions are going to happen becomes more certain.

Our method can perform robustly in the presence of noise (i.e. some detectors are weak and generate false positives or false negatives detections). In Figure 6, we show the result on the same sequence, only this time we completely disable the detectors of all actions involving bin 3 . There is great uncertainty at the beginning, but eventually the system is able to recognize the sequence of actions. The timings of bin 3's actions are estimated based upon the detection of other actions and the temporal constraint between them (e.g. body 3 is between body 2 and body4).

Quantitative Result: we can use the mean of the distributions as the timing estimation, and then the event localization error can be defined as the difference between this estimation and the true timing. Figure 7 shows how the result changes as more observation is available: the classification of the model being assembled (A, B or C) gets better, the average localization error of every actions' start time decreases, and the entropy of those distributions (representing the uncertainty) decreases. When the whole sequence has been seen, the average localization error is less than $1 \mathrm{sec}-$ ond. We also performed segmentation in offline mode (all observation is available) and the accuracy is $91.8 \%$.

Note that by taking the mean of the distributions, we are making a guess that minimizes the squared error. On the other hand, one can integrate a custom cost function over the distribution in order to make a prediction that minimizes the expected cost. In earlier work [7] we developed a linear-chain only method (no grammar) to apply in a Human Robot Collaboration context where the inference runs online in real-time. The robot was required to anticipate when a human would need a given part so that it could make a plan as to when to fetch bins and when to remove them. The goal was to create a plan that minimizes the human operator's idle time; in that situation the robot considered the entire timing distribution not just the mean.

\section{Recognition and Segmentation experiment}

We conducted two different activity segmentation experiments. In the first experiment, we constructed long sequences of multiple actions by concatenating short videos from Weizmann dataset [3]. Our method performed competitively in comparison to discriminative methods in [8] and [19]. Detail and result of this experiment is in the sup-
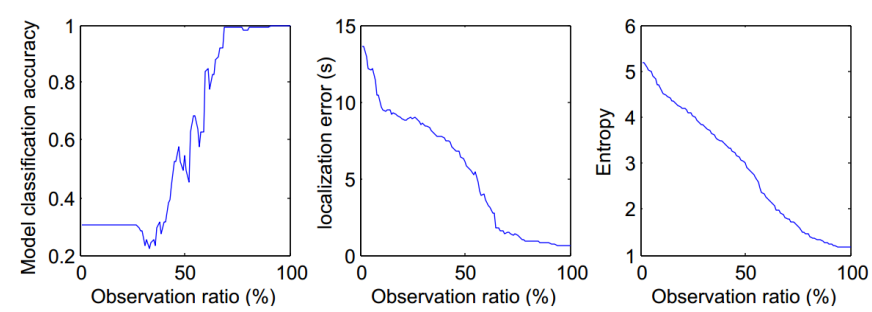

Figure 7. Result on our toy assembly dataset: (a) accuracy of model classification, (b) average localization error and (c) entropy of all actions' start

plemental document. In the following secton, we describe the second experiment, which is much more challenging.

\subsection{GeorgiaTech Egocentric Activity dataset}

The GeorgiaTech Egocentric Activity dataset (GTEA) [5,6] consists of 7 high level activities such as making a cheese sandwich or making coffee; each action is performed by 4 subjects. There are 61 primitives (such as take spoon, take cup, open sugar, scoop sugar spoon, open water, pour water, etc). Following [6], 16 sequences are used for training and 7 for testing.

For detection, we obtained the beginning state detection scores $S_{B}$ and ending state detection scores $S_{E}$ of every primitive from the author [6] (the classification accuracy is $39.7 \%$ ). Since these raw scores are not a good indicator of the likelihood, we define our detection score of a primitive $v$ as $F_{v}[\alpha, \beta] \propto\left(S_{B}[v, \alpha] S_{E}[v, \beta]\right)^{2}$ to magnify the difference between positive and negative detections. We also test a 2 nd setting, where we use $F_{v}[\alpha, \beta] \propto$ $\left(S_{B}[v, \alpha] S_{E}[v, \beta]\right)^{10}$.

The grammar is very important and design of a good grammar is not trivial (this will be discussed in next section). We derive our grammar using training sequences in a very simple way:

$$
\begin{aligned}
& S \rightarrow \text { Activity } 1 \mid \text { Activity } 2 \mid \ldots \\
& \text { Activity } 1 \rightarrow \text { Sequence } 1 \mid \text { Sequence } 2 \mid \ldots \\
& \text { Sequence } 1 \rightarrow \text { p_action } 1 \text { p_action } 2 \text { p_action } 3 \ldots
\end{aligned}
$$

This exemplar-like approach effectively matches the testing sequence with all the training data to find similar sequences (even though they are not exactly the same).

Our segmentation accuracy is $51 \%$ in the first detection score and 58\% in the 2nd setting, compare with [6]'s $42 \%$ and [5]'s 33\%. One example result is shown in Figure 8.

Unlike [6], our method models the global structure of the activity and is able to natively classify high level activity using posterior probabilities associated with the first ORrule. In this experiment, our method correctly classifies the high level activity label of 6 out of 7 test sequences.

\section{Conclusion}

We have presented a novel framework for modeling complex composite activity using a Stochastic Grammar. Pars- 


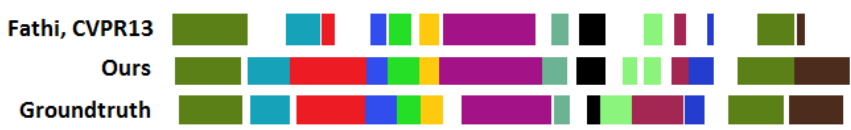

Figure 8. Example Segmentation result on GTEA of the activity: making Cheese Sandwich.

ing a sequence is done by performing message passing on the generated Bayes Network, called Sequential Interval Network. As shown in the experiments, our method outputs the posterior distributions of: (1) all actions' timing, which can be used for localization/prediction of actions/events, (2) strings realized by the grammar (the sequence of actions), which can be used for classifying high level activity or deriving sequence that best explains the observation, and (3) frames' label which can be used for activity segmentation.

For future work, we consider a dynamic time resolution approach for time series to speed up the inference speed. That way, even sequences with length of hours can be processed efficiently. While the grammar is flexible and can be constructed using expert's knowledge, designing a good grammar is not trivial. Moreover, it is desirable to learn it from training data, so the problem of grammar induction is very relevant to our work. Finally, the grammar can be extended to realize multiple streams of actions going on at the same time. This will be useful for modeling process such as complicated interaction between multiple agents.

\section{Acknowledgement}

We'd like to thank Alireza Fathi for providing data on GTEA dataset. This work was supported in part by BMW project \#RD441

\section{References}

[1] M. Albanese, R. Chellappa, V. Moscato, A. Picariello, V. Subrahmanian, P. Turaga, and O. Udrea. A constrained probabilistic petri net framework for human activity detection in video. Multimedia, IEEE Transactions on, 2008. 2

[2] M. R. Amer and S. Todorovic. Sum-product networks for modeling activities with stochastic structure. In CVPR, 2012. 2

[3] M. Blank, L. Gorelick, E. Shechtman, M. Irani, and R. Basri. Actions as space-time shapes. In ICCV, 2005. 7

[4] D. Damen and D. Hogg. Explaining activities as consistent groups of events. International journal of computer vision, 2012. 2

[5] A. Fathi, A. Farhadi, and J. M. Rehg. Understanding egocentric activities. In ICCV, 2011. 7

[6] A. Fathi and J. M. Rehg. Modeling actions through state changes. In $C V P R, 2013.7$

[7] K. P. Hawkins, N. Vo, S. Bansal, and A. Bobick. Probabilistic human action prediction and wait-sensitive planning for responsive human-robot collaboration. In Proceedings of the IEEE-RAS International Conference on Humanoid Robots, 2013. 7
[8] M. Hoai, Z.-Z. Lan, and F. De la Torre. Joint segmentation and classification of human actions in video. In CVPR, 2011. 2,7

[9] G. Hoffman and C. Breazeal. Cost-Based Anticipatory Action Selection for HumanRobot Fluency. IEEE Transactions on Robotics, 23(5):952-961, Oct. 2007. 1

[10] M. Huber and A. Knoll. When to assist?-Modelling human behaviour for hybrid assembly systems. In Robotics (ISR), 2010. 1

[11] Y. A. Ivanov and A. F. Bobick. Recognition of visual activities and interactions by stochastic parsing. Pattern Analysis and Machine Intelligence, IEEE Transactions on, 2000. 2

[12] D. Kollar and N. Friedman. Probabilistic graphical models: principles and techniques. The MIT Press, 2009. 4

[13] H. S. Koppula and A. Saxena. Learning spatio-temporal structure from rgb-d videos for human activity detection and anticipation. ICML, 2013. 2

[14] B. Laxton, J. Lim, and D. Kriegman. Leveraging temporal, contextual and ordering constraints for recognizing complex activities in video. In $C V P R, 2007.2$

[15] K. Li, J. Hu, and Y. Fu. Modeling complex temporal composition of actionlets for activity prediction. In ECCV. 2012. 2

[16] D. Moore and I. Essa. Recognizing multitasked activities from video using stochastic context-free grammar. In AAAI/IAAI, pages 770-776, 2002. 2

[17] K. P. Murphy. Hidden semi-markov models (hsmms). unpublished notes, 2002. 2

[18] M. Pei, Y. Jia, and S.-C. Zhu. Parsing video events with goal inference and intent prediction. In Computer vision (iccv), 2011 ieee international conference on, pages 487494. IEEE, 2011. 2

[19] Q. Shi, L. Wang, L. Cheng, and A. Smola. Discriminative human action segmentation and recognition using semimarkov model. In CVPR, 2008. 2, 7

[20] Y. Shi, Y. Huang, D. Minnen, A. Bobick, and I. Essa. Propagation networks for recognition of partially ordered sequential action. In $C V P R, 2004.2$

[21] Z. Si, M. Pei, B. Yao, and S.-C. Zhu. Unsupervised learning of event and-or grammar and semantics from video. In ICCV, 2011. 2

[22] K. Tang, L. Fei-Fei, and D. Koller. Learning latent temporal structure for complex event detection. In CVPR, 2012. 2

[23] H. Wang, A. Klaser, C. Schmid, and C.-L. Liu. Action recognition by dense trajectories. In CVPR, 2011. 2

[24] H. Wang, M. M. Ullah, A. Klaser, I. Laptev, C. Schmid, et al. Evaluation of local spatio-temporal features for action recognition. In $B M V C, 2009.2$

[25] P. Wei, Y. Zhao, N. Zheng, and S.-C. Zhu. Modeling 4d human-object interactions for event and object recognition. In $I C C V$, 2013. 2

[26] S.-Z. Yu. Hidden semi-markov models. Artificial Intelligence, 2010. 2 